\title{
Modelling and Solution of Infectious Diseases Using the Extended Laplace Adomian Decomposition Techniques
}

\author{
Bazuaye Frank Etin-Osa*, Ezeora Jeremiah \\ Department of Mathematics and Statistics, Faculty of Science, University of Port Harcourt, Port Harcourt, Nigeria
}

Email address:

febazuaye@yahoo.com (B. F. Etin-Osa)

${ }^{*}$ Corresponding author

\section{To cite this article:}

Bazuaye Frank Etin-Osa, Ezeora Jeremiah. Modelling and Solution of Infectious Diseases Using the Extended Laplace Adomian Decomposition Techniques. Applied and Computational Mathematics. Vol. 10, No. 2, 2021, pp. 30-39. doi: 10.11648/j.acm.20211002.11

Received: December 14, 2020; Accepted: January 21, 2021; Published: April 16, 2021

\begin{abstract}
The use of Mathematical models to describe the transmission of infectious diseases has attracted a lot of interest over the years and serious worldwide effort is accelerating the developments in the establishment of a global efforts for combating pandemics of infectious diseases. Scientists from different fields have teamed up for rapid assessment of potentially immediate situations. Toward this aim, mathematical modeling plays an important role in efforts that focus on predicting, assessing, and controlling potential outbreaks. The recent outbreak of covid 19 pandemic had increased the curiosity for the formulation of Mathematical models to describe and analyze the propagation of the disease. This paper focuses on the modeling and analysis of an infectious diseases model using the extended Laplace Adomian Decomposition (LAD) method. The method is used to obtain solutions in the form of infinite series. The result of the research with the aid of MAPLE indicates that physical contact with an infected person is the major cause of the propagation of any infectious disease in the absence of pharmaceutical and non pharmaceutical safety protocols such as the proper use of face mask, physical and social distancing. It becomes vital to subject the infected persons in isolation and adhere to the necessary protocols by relevance agencies and this will significantly flattened the curve of the spread of the infectious disease.
\end{abstract}

Keywords: Infectious Disease, Numerical Analysis, Mathematical Model, Susceptible Class, Infected Population

\section{Introduction}

$\mathrm{Wu}$ etal [1] modeling of infectious diseases is a potent instrument that has been used and is still been used to study the mechanisms by which diseases spread, to predict the future course of an outbreak and to evaluate strategies to control the epidemic. In other words, Rui-Zing et al. [2] opined that infectious diseases such as SARS and H1N1 can significantly impact people's lives and cause severe social and economic damages. Recent outbreaks have stressed the urgency of effective research on the dynamics of infectious disease spread. However, it is difficult to predict when and where outbreaks may emerge and how infectious diseases spread because many factors affect their transmission, and some of them may be unknown.

Several researchers had carried out studies on infectious diseases. Gurav et al. [3] reported about the influenza A/H1N1 2009 (Swine flu) outbreak in a residential school in Panchgani, Maharashtra. The epidemiologic data for the outbreak motivated Shil et al. $[4,5]$ to derived the intrinsic exponential growth rate for each day. On their part, Deguen et al. [6] analyzed the periodical pattern of chicken pox epidemic in France. The analysis was done by fitting SEIR model with a periodic contact rate function to weekly chicken pox incidence. Wang et al [7] adapted the SEIR model with a time dependant transmission rate (contact per infectious person per day) for describing the SARS outbreak in Beijing city. Kongnuy et al. [8] described a simple model for transmission of mosquito borne disease. In the model, the interaction between the infected mosquitoes and the uninfected humans will produce new infected humans. Constantenos et al [9], developed an agent-based model to investigate the epidemic dynamics of Ebola virus disease (EVD) in Liberia and Sierra Leo developed an agent-based model to investigate the epidemic dynamics of Ebola virus disease (EVD) in Liberia and Sierra Leon developed an agent-based model to investigate the epidemic dynamics of Ebola virus disease (EVD) in Liberia and Sierra Leon 
Ruan et al. [10] used the differential equations that described disease dynamics to analyze malaria and applied by Massad et al. [11] for description of Dengue transmission. The Laplace Adomian Decomposition Method (LADM) introduced by Adomian [12] provides an efficient method for finding the numerical solution of a system of differential equations models.

This method has been used in literature to solve differential and integral equations. It has been shown that the method significantly reduce the size of computational efforts while still retaining its accuracy, Adomian, Somali, Adomian, Wazwa [13-16]. The ADM decomposes a solution into an infinite series which converges rapidly to the exact solution.

\section{Materials and Methods}

In this section, the Mathematical model is developed. In order to successfully model this pandemic, the population was divided into four sectors They are: the susceptible, infected and the recovered from the disease.

$$
\left.\begin{array}{l}
\frac{d S(t)}{d t}=B-\theta S(t) I(t)-\tau S(t), \\
\frac{d I(t)}{d t}=\theta S(t) I(t)-\psi I(t)-\tau I(t) \\
\frac{d R(t)}{d t}=\psi I(t)
\end{array}\right\}
$$

With the initial conditions

$$
S(0)=S_{0} \geq 0, I(0)=I_{0} \geq 0, R(0)=R_{0} \geq 0,
$$

Where the parameters are defined as follows

$S$ is the susceptible population

$I$ is the infected population

$R$ is the recovered population from the pandemic

$\theta$ is the mean transmission rate of the infectious disease

$\psi$ is the mean recovery rate

$\tau$ represents the mortality rate per capital

$B$ Shows the birth rate per unit time

Under the initial conditions (2) all the solution $S(t) ., I(t)$ and $R(t)$ of system (1) remain nonnegative for all positive $\mathrm{t}$

\subsection{The Extended Laplace-Adomian Decomposition Method}

The numerical solution of the proposed mode is obtained by the Adomian Decomposition method. The Laplace transformation is used to convert the system of differential equations into a system of algebraic equations. Then, the algebraic equations are used to obtain the required solution in form of series. We will discuss the procedure for solving model (1) with given initial conditions (2). Applying Laplace transform on both sides of model (1), we obtain the following system:

$$
\begin{aligned}
& \ell\left\{\frac{d S(t)}{d t}\right\}=\ell\{B-\theta S(t) I(t)-\tau S(t)\}, \\
& \ell\left\{\frac{d I(t)}{d t}\right\}=\ell\{\theta S(t) I(t)-\psi I(t)-\tau I(t)\}, \\
& \ell\left\{\frac{d R(t)}{d t}\right\}=\ell\{\psi I(t)\}
\end{aligned}
$$

Equations (3) result

$$
\left.\begin{array}{l}
s \ell\{S(t)\}-S(0)=\ell\{B-\theta S(t) I(t)-\tau S(t)\}, \\
s \ell\{I(t)\}-I(0)=\ell\{\theta S(t) I(t)-\psi I(t)-\tau I(t)\}, \\
s \ell\{R(t)\}-R(0)=\ell\{\psi I(t)\}
\end{array}\right\}
$$

Simplifying (4) by applying the initial conditions (2) results

$$
\left.\begin{array}{l}
\ell\{S(t)\}=\frac{S(0)}{s}+\left[\frac{\ell\{B-\theta S(t) I(t)-\tau S(t)\}}{s}\right] \\
s \ell\{I(t)\}=\frac{I(0)}{s}+\left[\frac{\ell\{\theta S(t) I(t)-\psi I(t)-\tau I(t)\}}{s}\right] \\
s \ell\{R(t)\}=\frac{R(0)}{s}+\ell \frac{\{\psi I(t)\}}{s}
\end{array}\right\}
$$

Now, assume that the solutions $S(t) ., I(t)$ and $R(t)$ are in the form of infinite series given by

$$
\left.\begin{array}{l}
S(t)=\sum_{\eta=0}^{\infty} S_{\eta}(t) \\
I(t)=\sum_{\eta=0}^{\infty} I_{\eta}(t) \\
R(t)=\sum_{\eta=0}^{\infty} R_{\eta}(t)
\end{array}\right\}
$$

Decomposing the nonlinear term $S(t) I(t)$ as

$$
\left.S(t) I(t)=\sum_{i=0}^{\infty} M_{i}(t)\right\}
$$

Respectively.

Where each $M_{\eta}$ is the Adomian polynomials defined as

$$
\left.M_{\eta}=\frac{1}{\eta !} \frac{d^{\eta}}{d \lambda^{\eta}} \mid \sum_{\zeta=0}^{\eta} \lambda^{\zeta} S_{\lambda}(t) \sum_{\zeta=0}^{\eta} \lambda^{\zeta} I_{\lambda}(t) \|_{\lambda=0},\right\}
$$

Expanding (7) yields the following polynomials

$$
\begin{aligned}
& M_{0}=S_{O}(t) I_{o}(t) \\
& M_{1}=\left.\frac{d}{d \lambda}\left[\left(\lambda^{0} S_{0}(t)+\lambda^{1} S_{1}(t)\right)\left(\lambda^{0} I_{0}(t)+\lambda^{1} I_{1}(t)\right)\right]\right|_{\lambda=0} \\
& \frac{d}{d \lambda}\left[\left(S_{0}(t)+\lambda S_{1}(t)\right)\left(I_{0}(t)+\lambda I_{1}(t)\right)\right]_{\lambda=0}
\end{aligned}
$$


Expanding (9) yields

$\left.\frac{d}{d \lambda}\left[\left(S_{0}(t) I_{0}(t)+\lambda S_{0}(t)\right)\left(I_{1}(t)+\lambda S_{1} I_{0}(t)+\lambda^{2} S_{1} I_{1}(t)\right)\right]\right|_{\lambda=0}(10)$

$Z_{1}=S_{0}(t) I_{1}(t)+S_{1}(t) I_{0}(t)$

Similarly,

$$
Z_{2}=2 S_{0}(t) I_{2}(t)+2 S_{1}(t) I_{1}(t)+2 S_{2}(t) I_{0}(t)
$$

Substituting (6) into (5) results in

$\ell\{S(t)\}=\frac{S(0)}{s}+\left[\frac{\ell\left\{B-\theta \sum_{\eta}^{\infty} M_{\eta}(t)-\tau \sum_{\eta}^{\infty} S_{\eta}(t)\right\}}{s^{\gamma}}\right]$

$\left.s \ell\{I(t)\}=\frac{I(0)}{s}+\left[\frac{\ell\left\{\theta \sum_{\eta}^{\infty} M_{\eta}(t)-\psi \sum_{\eta}^{\infty} I_{\eta}(t)-\tau \sum_{\eta}^{\infty} I_{\eta}(t)\right\}}{s^{\gamma}}\right]\right\}$

$s \ell\{R(t)\}=\frac{R(0)}{s}+\ell \frac{\left\{\psi \sum_{\eta}^{\infty} I_{\eta}(t)\right\}}{s^{\gamma}}$

Comparing the two sides of equation (12) results the following iterative algorithm

$$
\begin{aligned}
& \ell\{S(t)\}=\frac{S(0)}{s}+\left[\frac{\ell\left\{B-\theta \sum_{\eta}^{\infty} M_{\eta}(t)-\tau \sum_{\eta}^{\infty} S_{\eta}(t)\right\}}{s^{\gamma}}\right] \\
& \left.s \ell\{I(t)\}=\frac{I(0)}{s}+\left[\frac{\ell\left\{\theta \sum_{\eta}^{\infty} M_{\eta}(t)-\psi \sum_{\eta}^{\infty} I_{\eta}(t)-\tau \sum_{\eta}^{\infty} I_{\eta}(t)\right\}}{\left.s^{\gamma}\right\}}\right]\right\} \\
& s \ell\{R(t)\}=\frac{R(0)}{s}+\ell \frac{\left\{\psi \sum_{\eta}^{\infty} I_{\eta}(t)\right\}}{s^{\gamma}}
\end{aligned}
$$

$$
\left.\begin{array}{l}
\ell\left\{S_{0}\right\}=\frac{N_{1}}{s} \\
\ell\left\{S_{1}\right\}=\frac{B}{s^{\gamma}}-\frac{\theta}{s^{\gamma}} \ell\left\{M_{0}\right\}-\frac{\tau}{s^{\gamma}} \ell\left\{S_{0}\right\} \\
\ell\left\{S_{2}\right\}=\frac{B}{s^{\gamma}}-\frac{\theta}{s^{\gamma}} \ell\left\{M_{1}\right\}-\frac{\tau}{s^{\gamma}} \ell\left\{S_{1}\right\} \\
\ell\left\{S_{3}\right\}=\frac{B}{s^{\gamma}}-\frac{\theta}{s^{\gamma}} \ell\left\{M_{2}\right\}-\frac{\tau}{s^{\gamma}} \ell\left\{S_{2}\right\} \\
\vdots \\
\ell\left\{S_{d+1}\right\}=\frac{B}{s^{\gamma}}-\frac{\theta}{s^{\gamma}} \ell\left\{M_{0}\right\}-\frac{\tau}{s^{\gamma}} \ell\left\{S_{d}\right\}
\end{array}\right\}
$$

Similarly,

$$
\begin{aligned}
& \ell\left\{I_{0}\right\}=\frac{N_{2}}{s} \\
& \ell\{I(t)\}=\frac{I(0)}{s}+\left[\frac{\ell\left\{\theta \sum_{\eta}^{\infty} M_{\eta}(t)-\psi \sum_{\eta}^{\infty} I_{\eta}(t)-\tau \sum_{\eta}^{\infty} I_{\eta}(t)\right\}}{s^{\gamma}}\right] \\
& \ell\left\{I_{0}\right\}=\frac{N_{2}}{s} \\
& \ell\left\{I_{1}\right\}=\frac{\theta}{s^{\gamma}} \ell\left\{M_{0}\right\}-\frac{(\psi+\tau)}{s^{\gamma}} \ell\left\{I_{0}\right\} \\
& \ell\left\{I_{2}\right\}=\frac{\theta}{s^{\gamma}} \ell\left\{M_{1}\right\}-\frac{(\psi+\tau)}{s^{\gamma}} \ell\left\{I_{1}\right\} \\
& \ell\left\{I_{3}\right\}=\frac{\theta}{s^{\gamma}} \ell\left\{M_{2}\right\}-\frac{(\psi+\tau)}{s^{\gamma}} \ell\left\{I_{2}\right\} \\
& \vdots \\
& \ell\left\{I_{d+1}\right\}=\frac{\theta}{s^{\gamma}} \ell\left\{M_{d}\right\}-\frac{(\psi+\tau)}{s^{\gamma}} \ell\left\{I_{d}\right\}
\end{aligned}
$$

\subsection{Application of The Extended Laplace-Adomian Decomposition Method}

In this session, the extended Laplace Decomposition Method is applied to the infectious diseases model

$$
\left.\begin{array}{l}
\ell\left\{R_{0}\right\}=\frac{N_{3}}{s} \\
\ell\left\{R_{1}\right\}=\frac{\psi}{s^{\gamma}} \ell\left\{I_{0}\right\} \\
\ell\left\{R_{2}\right\}=\frac{\psi}{s^{\gamma}} \ell\left\{I_{1}\right\} \\
\ell\left\{R_{3}\right\}=\frac{\psi}{s^{\gamma}} \ell\left\{I_{2}\right\} \\
\vdots \\
\ell\left\{R_{d+1}\right\}=\frac{\psi}{s^{\gamma}} \ell\left\{I_{d}\right\}
\end{array}\right\}
$$

Evaluating the inverse Laplace transform of (13), (14) and (15) and considering the first few terms yields

$$
\left.S_{1}=\frac{t^{\gamma}}{\Gamma(\gamma+1)} B-\frac{t^{\gamma}}{\Gamma(\gamma+1)} \theta M_{0}-\tau \frac{t^{\gamma}}{\Gamma(\gamma+1)} S_{0}\right\}
$$

With the following parameters defined as follows

$$
\begin{aligned}
& S_{0}=N_{1} \\
& I_{0}=N_{2} \\
& R_{0}=N_{3} \\
& M_{0}=S_{0} I_{0}
\end{aligned}
$$

Substituting 


$$
\left.S_{1}=\frac{t^{\gamma}}{\Gamma(\gamma+1)} B-\frac{t^{\gamma}}{\Gamma(\gamma+1)} N_{1} N_{2}-\tau \frac{t^{\gamma}}{\Gamma(\gamma+1)} N_{1}\right\}
$$

Similarly, $S_{2}$ and $S_{3}$ are

$$
\begin{aligned}
& S_{2}=\frac{t^{\gamma}}{\Gamma(\gamma+1)} B-\frac{1}{\Gamma(\gamma+1)}\left(\frac{t^{\gamma} \theta\left(\left(N_{2}+\frac{t^{\gamma} \theta N_{1} N_{2}}{\Gamma(\gamma+1)}-\frac{t^{\gamma}(\psi+\tau)}{\Gamma(\gamma+1)} N_{2}\right) N_{1}\right.}{\Gamma(\gamma+1)}\right. \\
& \left.+N_{2}\left(\frac{t^{\gamma}}{\Gamma(\gamma+1)} B-\frac{t^{\gamma}}{\Gamma(\gamma+1)} \theta N_{1} N_{2}-\frac{t^{\gamma}}{\Gamma(\gamma+1)} N_{1}\right) \frac{-t^{\gamma}\left(\frac{t^{\gamma} B}{\Gamma(\gamma+1)}-\frac{t^{\gamma} \theta N_{1} N_{2}}{\Gamma(\gamma+1)}-\frac{t^{\gamma} N_{1}}{\Gamma(\gamma+1)}\right)}{\Gamma(\gamma+1)}\right) \\
& S_{3}=\frac{1}{\Gamma(\gamma+1)}\left(\frac{t^{\gamma} \theta\left(2 N _ { 2 } \left(\frac{t^{\gamma} B}{\Gamma(\gamma+1)}-\frac{1}{\Gamma(\gamma+1)}\left(t ^ { \gamma } \psi \left(\left(N_{2}+\frac{t^{\gamma}\left(\psi N_{1} N_{2}\right)}{\Gamma(\gamma+1)} N_{2}-\frac{t^{\gamma}(\psi+\tau) N_{2}}{\Gamma(\gamma+1)}\right) N_{1}\right.\right.\right.\right.}{\Gamma(\gamma+1)}-\right. \\
& \frac{1}{\Gamma(\gamma+1)}\left(\frac{t^{\gamma} \theta\left(2 N _ { 2 } \left(\frac{t^{\gamma} B}{\Gamma(\gamma+1)}-\frac{1}{\Gamma(\gamma+1)}\left(t ^ { \gamma } \psi \left(\left(N_{2}+\frac{t^{\gamma}\left(\psi N_{1} N_{2}\right)}{\Gamma(\gamma+1)} N_{2}-\frac{t^{\gamma}(\psi+\tau) N_{2}}{\Gamma(\gamma+1)}\right) N_{1}\right.\right.\right.\right.}{\Gamma(\gamma+1)}\right. \\
& +N_{2}\left(\frac{t^{\gamma}}{\Gamma(\gamma+1)} B-\frac{t^{\gamma}}{\Gamma(\gamma+1)} \theta N_{1} N_{2}-\frac{t^{\gamma}}{\Gamma(\gamma+1)} N_{1}\right)^{-t^{\gamma}\left(\frac{t^{\gamma} B}{\Gamma(\gamma+1)}-\frac{t^{\gamma} \theta N_{1} N_{2}}{\Gamma(\gamma+1)}-\frac{t^{\gamma} N_{1}}{\Gamma(\gamma+1)}\right)}+2\left(N_{2}+\right. \\
& \left.\left.\left.\frac{1}{\Gamma(\gamma+1)} \frac{t^{\gamma} \theta\left(\left(N_{2}+\frac{t^{\gamma} \theta N_{1} N_{2}}{\Gamma(\gamma+1)}-\frac{t^{\gamma}(\psi+\tau) N_{2}}{\Gamma(\gamma+1)}\right) N_{1}+N_{2}\left(\frac{t^{\gamma}}{\Gamma(\gamma+1)} B-\right.\right.}{\Gamma(\gamma+1)} \frac{t^{\gamma} \theta N_{1} N_{2}}{\Gamma(\gamma+1)}-\frac{t^{\gamma}}{\Gamma(\gamma+1)} N_{1}\right)\right)\right)- \\
& \frac{\left.\left.\frac{t^{\gamma} \theta N_{1} N_{2}}{\Gamma(\gamma+1)}-\frac{t^{\gamma}(\psi+\tau) N_{2}}{\Gamma(\gamma+1)}\right) N_{1}\right)-\frac{1}{\Gamma(\gamma+1)}\left(t^{\gamma}\left(\frac{t^{\gamma}}{\Gamma(\gamma+1)} B-\frac{1}{\Gamma(\gamma+1)}\right) t^{\gamma} \theta\right)\left(N_{2}+\frac{t^{\gamma} \theta N_{1} N_{2}}{\Gamma(\gamma+1)}-\frac{t^{\gamma}(\psi+\tau) N_{2}}{\Gamma(\gamma+1)}\right) N_{1}+}{\Gamma(\gamma+1)} \\
& \frac{N_{2}\left(\frac{t^{\gamma}}{\Gamma(\gamma+1)} B-\frac{t^{\gamma} \theta N_{1} N_{2}}{\Gamma(\gamma+1)}-\frac{t^{\gamma} N_{1}}{\Gamma(\gamma+1)}\right)-t^{\gamma}\left(\frac{t^{\gamma} B}{\Gamma(\gamma+1)}-\frac{t^{\gamma} \theta N_{1} N_{2}}{\Gamma(\gamma+1)}-\frac{t^{\gamma} N_{1}}{\Gamma(\gamma+1)}\right)}{\Gamma(\gamma+1)}
\end{aligned}
$$

For the infected class

$$
I_{1}=N_{2}+\frac{t^{\gamma} \theta N_{1} N_{2}}{\Gamma(\gamma+1)}-\frac{t^{\gamma}(\psi+\tau) N_{2}}{\Gamma(\gamma+1)}
$$




$$
\begin{gathered}
I_{2}=N_{2}+\frac{\frac{t^{\gamma}}{\Gamma(\gamma+1)}\left(t^{\gamma} \theta\left(\left(N_{2}+\frac{t^{\gamma} \theta N_{1} N_{2}}{\Gamma(\gamma+1)}-\frac{t^{\gamma}(\psi+\tau) N_{2}}{\Gamma(\gamma+1)}\right) N_{1}+N_{2}\right) \frac{t^{\gamma} B}{\Gamma(\gamma+1)}\right)-\frac{t^{\gamma} \theta N_{1} N_{2}}{\Gamma(\gamma+1)}}{\Gamma(\gamma+1)} \\
-\frac{t^{\gamma} N_{1}}{\Gamma(\gamma+1)}-t^{\gamma}(\psi+\tau)\left(N_{2}+\frac{t^{\gamma} \theta N_{1} N_{2}}{\Gamma(\gamma+1)}-\frac{t^{\gamma}(\psi+\tau) N_{2}}{\Gamma(\gamma+1)}\right) N_{2} \\
I_{3}=N_{2}+\frac{1}{\Gamma(\gamma+1)}\left(t ^ { \gamma } \theta \left(2 N_{2}\left(\frac{t^{\gamma} B}{\Gamma(\gamma+1)}-\frac{1}{\Gamma(\gamma+1)}\left(t^{\gamma} \theta\right) N_{2}+\frac{t^{\gamma} \theta N_{1} N_{2}}{\Gamma(\gamma+1)}-\frac{t^{\gamma}(\psi+\tau) N_{2}}{\Gamma(\gamma+1)}\right) N_{1}\right.\right. \\
N_{2}\left(\left(\frac{t^{\gamma} B}{\Gamma(\gamma+1)}-\frac{t^{\gamma} \theta N_{1} N_{2}}{\Gamma(\gamma+1)}-\frac{t^{\gamma} N_{1}}{\Gamma(\gamma+1)}\right)\right)-\frac{t^{\gamma}\left(\frac{t^{\gamma} B}{\Gamma(\gamma+1)}-\frac{t^{\gamma} \theta N_{1} N_{2}}{\Gamma(\gamma+1)}-\frac{t^{\gamma} N_{1}}{\Gamma(\gamma+1)}\right)}{\Gamma(\gamma+1)} \\
+2\left(N_{2}+\frac{t^{\gamma} \theta N_{1} N_{2}}{\Gamma(\gamma+1)}-\frac{t^{\gamma}(\psi+\tau) N_{2}}{\Gamma(\gamma+1)}\right)\left(\frac{t^{\gamma} B}{\Gamma(\gamma+1)}-\frac{t^{\gamma} \theta N_{1} N_{2}}{\Gamma(\gamma+1)}-\frac{t^{\gamma} N_{1}}{\Gamma(\gamma+1)}\right) \\
+\frac{N_{2}\left(\frac{t^{\gamma} B}{\Gamma(\gamma+1)}-\frac{t^{\gamma} \theta N_{1} N_{2}}{\Gamma(\gamma+1)}-\frac{t^{\gamma} N_{1}}{\Gamma(\gamma+1)}-\right)}{\Gamma(\gamma+1)} \frac{t^{\gamma}(\psi+\tau)\left(N_{2}+\frac{t^{\gamma} \theta N_{1} N_{2}}{\Gamma(\gamma+1)}-\frac{t^{\gamma}(\psi+\tau) N_{2}}{\Gamma(\gamma+1)}\right) N_{1}}{\Gamma(\gamma+1)} \\
+\frac{t^{\gamma}(\psi+\tau)\left(N_{2}+\frac{t^{\gamma} \theta N_{1} N_{2}}{\Gamma(\gamma+1)}-\frac{t^{\gamma}(\psi+\tau) N_{2}}{\Gamma(\gamma+1)}\right) N_{1}-\frac{1}{\Gamma(\gamma+1)}\left(t^{\gamma}(\psi+\tau)\left(N_{2}+\frac{t^{\gamma} \theta N_{1} N_{2}}{\Gamma(\gamma+1)}-\frac{t^{\gamma}(\psi+\tau) N_{2}}{\Gamma(\gamma+1)}\right) N_{1}\right)}{\Gamma(\gamma+1)}\left(t^{\gamma} \theta\left(N_{2}+\frac{t^{\gamma} \theta N_{1} N_{2}}{\Gamma(\gamma+1)}-\frac{t^{\gamma}(\psi+\tau) N_{2}}{\Gamma(\gamma+1)}\right) N_{1}+N_{2}\left(\frac{t^{\gamma} B}{\Gamma(\gamma+1)}-\frac{t^{\gamma} \theta N_{1} N_{2}}{\Gamma(\gamma+1)}-\frac{t^{\gamma} N_{1}}{\Gamma(\gamma+1)}\right)\right) \\
\left.-\frac{1}{\Gamma(\gamma+1)}\right)
\end{gathered}
$$

Finally, for the recovered class $\mathrm{R}(\mathrm{t})$

$$
\begin{aligned}
& R_{1}=N_{3}+\frac{t^{\gamma}}{\Gamma(\gamma+1)} N_{2} \\
& R_{2}=N_{3}+\frac{t^{\gamma}\left(N_{2}+\frac{t^{\gamma} \theta N_{1} N_{2}}{\Gamma(\gamma+1)}-\frac{t^{\gamma}(\psi+\tau) N_{2}}{\Gamma(\gamma+1)}\right)}{\Gamma(\gamma+1)} \\
& R_{3}=N_{3}+\frac{1}{\Gamma(\gamma+1)}\left(t ^ { \gamma } \left(N_{2}+\frac{1}{\Gamma(\gamma+1)}\left(t^{\gamma} \theta\left(\left(N_{2}+\frac{t^{\gamma} \theta N_{1} N_{2}}{\Gamma(\gamma+1)}-\frac{t^{\gamma}(\psi+\tau) N_{2}}{\Gamma(\gamma+1)}\right) N_{1}\right)\right)\right.\right. \\
& \left.+N_{2}\left(\frac{t^{\gamma} B}{\Gamma(\gamma+1)}-\frac{t^{\gamma} \theta N_{1} N_{2}}{\Gamma(\gamma+1)}-\frac{t^{\gamma} N_{1}}{\Gamma(\gamma+1)}\right)-\frac{t^{\gamma}(\psi+\tau)\left(N_{2}+\frac{t^{\gamma} \theta N_{1} N_{2}}{\Gamma(\gamma+1)}-\frac{t^{\gamma}(\psi+\tau) N_{2}}{\Gamma(\gamma+1)}\right)}{\Gamma(\gamma+1)}\right)
\end{aligned}
$$




\section{Numerical Method and Results}

In this session, the efficiency of the proposed method is demonstrated. Given

$$
\begin{aligned}
& S_{0}=N_{1}=1, I_{0}=N_{2}=0.2, R_{0}=N_{3}=0.5, B=100, \tau=0.01, \theta=0.9, \\
& \psi=0.02
\end{aligned}
$$

The proposed Laplace Adomian Decomposition method for analyzing the Covid 19 Mathematical Model provides us with a numerical solution in the form of an infinite series

$$
\left.S(m)=N_{0}+\frac{{ }^{\gamma}}{\Gamma(\gamma+1)} B-\frac{{ }^{\gamma}}{\Gamma(\gamma+1)} \theta M_{2}-\tau \frac{t^{\gamma}}{\Gamma(\gamma+1)} S_{2}\right\}
$$

$$
\begin{aligned}
& \gamma=0.1 \\
& S(r)=105.137006 t^{0.1}-0.9460233055\left(t^{0.1}\left(207.6836496 t^{0.1}\left(0.2+0.1828978391 t^{0.1}\right)+0.4+1.892046611 t^{0.1}\right)\right) \\
& \left.\left.\left(0.2+20.95756962 t^{0 / 1}\right)+42.04548024 t^{0.1}-0.3784093222\left(\left(t^{0.1}\right)\left(0.2+20.95756962 t^{0.1}\right)\right)-0.4367405260 t^{0.2}\right)\right) \\
& -0.01051137006 t^{0.1}\left(1051.1137006 t^{0.1}-0.9460233055\left(\left(t^{0.1}\right)\left(0.2+20.95756962 t^{0.1}\right)-11.03585236 t^{0.2}\right.\right. \\
& \gamma=0.2 \\
& S(t)=1089.124421 t^{0.2}-0.9802119790\left(t^{0.2}\left(207.6836496 t^{0.1}\left(0.2+0.1828978391 t^{0.1}\right)+0.4+1.892046611 t^{0.1}\right)\right) \\
& \left.\left.\left(0.2+20.95756962 t^{0 / 1}\right)+42.04548024 t^{0.1}-0.3784093222\left(\left(t^{0.1}\right)\left(0.2+20.95756962 t^{0.1}\right)\right)-0.4367405260 t^{0.2}\right)\right) \\
& \left.-0.01089124421 t^{0.2}\left(1051.37006 t^{0.1}-0.9460233055\left(\left(t^{0.1}\right) 0.2+20.95756962 t^{0.1}\right)\right)-11.03585236 t^{0.2}\right) \\
& \gamma=0.3 \\
& S(u)=1114.242509 t^{0.3}-1.002818258\left(t^{0.3}\left(207.6836496 t^{0.1}\left(0.2+0.1828978391 t^{0.1}\right)+0.4+1.892046611 t^{0.1}\right)\right) \\
& \left.\left.\left(0.2+20.95756962 t^{0 / 1}\right)+42.04548024 t^{0.1}-0.3784093222\left(\left(t^{0.1}\right)\left(0.2+20.95756962 t^{0.1}\right)\right)-0.4367405260 t^{0.2}\right)\right) \\
& \left.-0.01114242509 t^{0.3}\left(1051.137006 t^{0.1}-0.9460233055\left(\left(t^{0.1}\right) 0.2+20.95756962 t^{0.1}\right)\right)-11.03585236 t^{0.2}\right) \\
& \gamma=0.4 \\
& S(v)=1127.060498 t^{0.4}-1.01435448\left(t^{0.4}\left(207.6836496 t^{0.1}\left(0.2+0.1828978391 t^{0.1}\right)+0.4+1.892046611 t^{0.1}\right)\right) \\
& \left.\left.\left(0.2+20.95756962 t^{0 / 1}\right)+42.04548024 t^{0.1}-0.3784093222\left(\left(t^{0.1}\right)\left(0.2+20.95756962 t^{0.1}\right)\right)-0.4367405260 t^{0.2}\right)\right) \\
& \left.-0.01127060498 t^{0.4}\left(1051.137006 t^{0.1}-0.9460233055\left(\left(t^{0.1}\right) 0.2+20.95756962 t^{0.1}\right)\right)-11.03585236 t^{0.2}\right) \\
& \gamma=0.5 \\
& S(w)=1128.379167 t^{0.5}-1.015541250\left(t^{0.5}\left(207.6836496 t^{0.1}\left(0.2+0.1828978391 t^{0.1}\right)+0.4+1.892046611 t^{0.1}\right)\right) \\
& \left.\left.\left(0.2+20.95756962 t^{0 / 1}\right)+42.04548024 t^{0.1}-0.3784093222\left(\left(t^{0.1}\right)\left(0.2+20.95756962 t^{0.1}\right)\right)-0.4367405260 t^{0.2}\right)\right) \\
& \left.-0.01128379167 t^{0.5}\left(105.137006 t^{0.1}-0.9460233055\left(\left(t^{0.1}\right) 0.2+20.95756962 t^{0.1}\right)\right)-11.03585236 t^{0.2}\right)
\end{aligned}
$$

The plot of $S(t)$ for $\gamma=0.1=S_{r} ; \gamma=0.2=S_{t} ; \gamma=0.3=S_{u} ; \gamma=0.4=S_{v} ; \gamma=0.5=S_{w}$ 


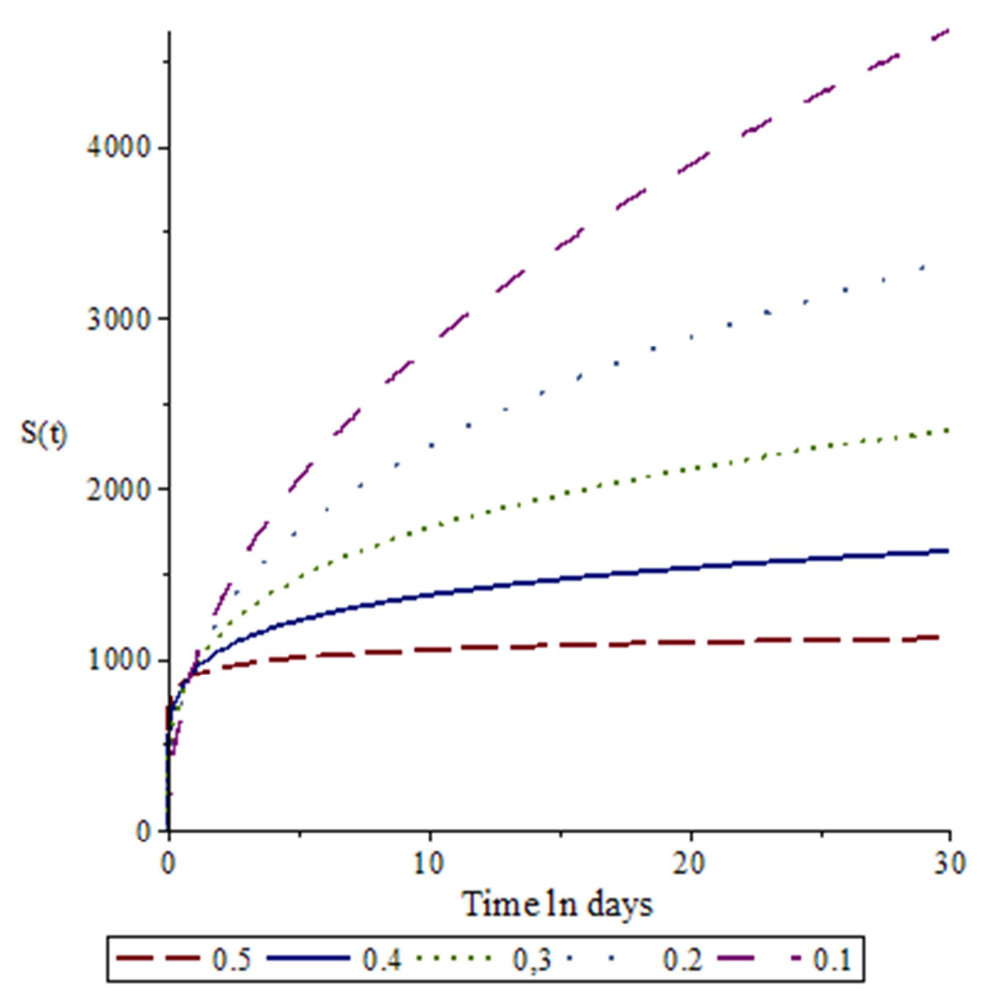

Figure 1. Plot of numerical solution of susceptible class $S(t)$ corresponding to different timein a day.

For the infected group;

$$
I(t)=N_{2}+\frac{t^{\gamma} \theta M_{2}}{\Gamma(\gamma+1)}-\frac{t^{\gamma}(\psi+\tau) I_{2}}{\Gamma(\gamma+1)}
$$

$\gamma=0.1$

$I(r)=0.2+0.94023305 t^{0.1}\left(42.0454804 t^{0.1}-29.7170946 t^{0.2}+207.95694\left(0.2+0.05886367 t^{0.1}\right) t^{0.1}+0.4\right)$

$-0.03153411018 t^{0.1}\left(0.2+0.9460233055 t^{0.1}\right)\left(20.85455819 t^{0.1}+0.2\right)-0.03153411018$

$\left.\left(0.2+0.058886367234 t^{0.1}\right) t^{0.1}\right)$

$\gamma=0.2$

$I(s)=0.2+0.9802119790 t^{0.2}\left(42.0454804 t^{0.1}-29.7170946 t^{0.2}+207.95694\left(0.2+0.05886367 t^{0.1}\right) t^{0.1}+0.4\right)$

$-0.03267373263 t^{0.2}\left(0.2+0.9460233055 t^{0.1}\left(20.85455819 t^{0.1}+0.2\right)-0.03153411018\right.$

$\left.\left(0.2+0.05886367234 t^{0.1}\right) t^{0.1}\right)$

$\gamma=0.3$

$I(u)=0.2+1.002818258 t^{0.3}\left(42.0454804 t^{0.1}-29.7170946 t^{0.2}+207.95694\left(0.2+0.05886367 t^{0.1}\right) t^{0.1}+0.4\right)$

$-0.033422727526 t^{0.3}\left(0.2+0.9460233055 t^{0.1}\left(20.85455819 t^{0.1}+0.2\right)-0.03153411018\right.$

$\left.\left(0.2+0.05886367234 t^{0.1}\right) t^{0.1}\right)$ 


$$
\begin{aligned}
& \gamma=0.4 \\
& I(v)=0.2+1.014354448 t^{0.4}\left(42.0454804 t^{0.1}-29.7170946 t^{0.2}+207.95694\left(0.2+0.05886367 t^{0.1}\right) t^{0.1}+0.4\right) \\
& -0.03381181494 t^{0.4}\left(0.2+0.9460233055 t^{0.1}\left(20.85455819 t^{0.1}+0.2\right)-0.03153411018\right. \\
& \left.\left(0.2+0.05886367234 t^{0.1}\right) t^{0.1}\right) \\
& \gamma=0.5 \\
& I(w)=0.2+1.015541250 t^{0.5}\left(42.0454804 t^{0.1}-29.7170946 t^{0.2}+207.95694\left(0.2+0.05886367 t^{0.1}\right) t^{0.1}+0.4\right) \\
& -0.0338513750 t^{0.5}\left(0.2+0.9460233055 t^{0.1}\left(20.85455819 t^{0.1}+0.2\right)-0.03153411018\right. \\
& \left.\left(0.2+0.05886367234 t^{0.1}\right) t^{0.1}\right)
\end{aligned}
$$

The plot of $I(t)$ for $\gamma=0.1=I_{r} ; \gamma=0.2=I_{s} ; \gamma=0.3=l_{u} ; \gamma=0.4=l_{v} ; \gamma=0.5=l_{w}$ Is shown the figure 2 below

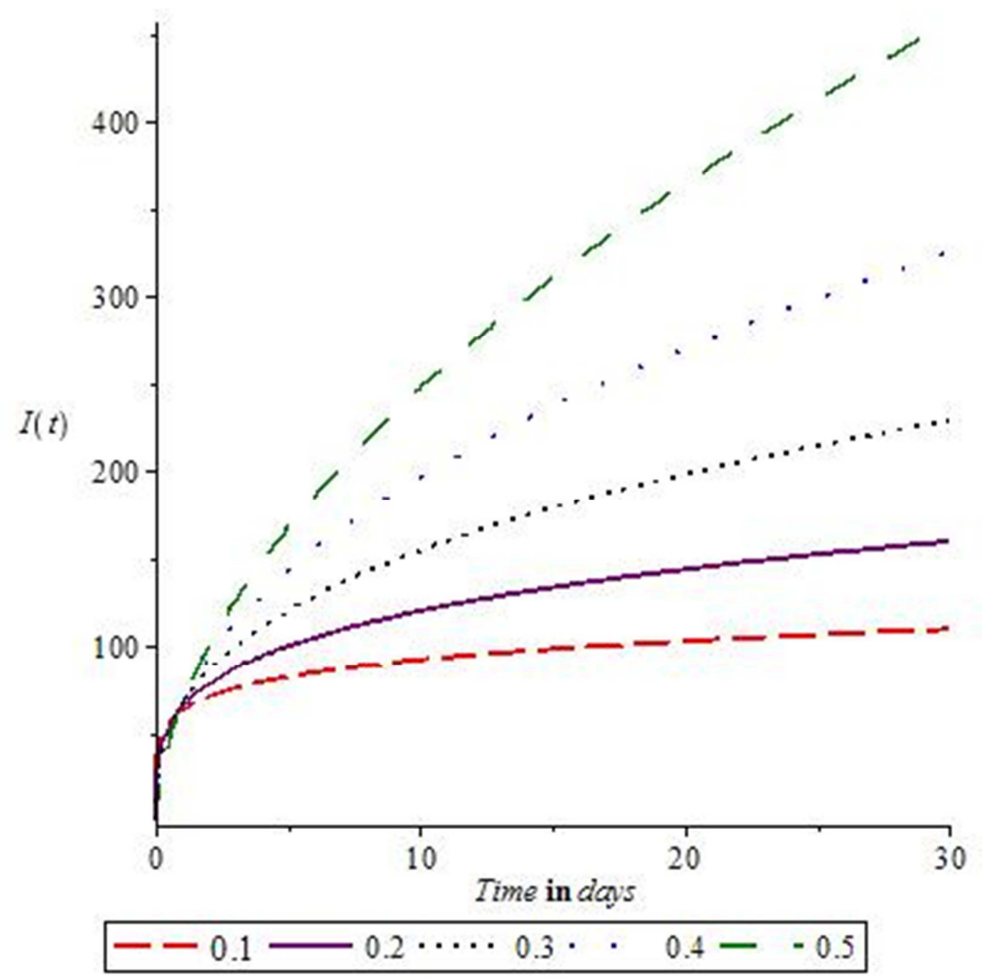

Figure 2. Plot of numerical solution of infected class I(t) corresponding to different timein a days.

Finally, the analysis of the recovered class is given below $0.4+0.04405 t^{2}$.

$$
\left.R(t)=N_{3}+\frac{t^{\gamma} \psi}{\Gamma(\gamma+1)} I_{2}\right\}
$$

$$
\begin{aligned}
& \gamma=0.1 \\
& R(r)=0.5+0.02102274012 t^{0.1}\left(0.2+0.9460233055 t^{0.1}\left(20.85455819 t^{0.1}+0.2\right)\right. \\
& \left.-0.03153411018\left(0.2+0.05886367234 t^{0.1}\right) t^{0.1}\right)
\end{aligned}
$$




$$
\begin{aligned}
& \gamma=0.2 \\
& R(s)=0.5+0.02178248842 t^{0.2}\left(0.2+0.9460233055 t^{0.1}\left(20.85455819 t^{0.1}+0.2\right)\right. \\
& \left.-0.03153411018\left(0.2+0.05886367234 t^{0.1}\right) t^{0.1}\right) \\
& \gamma=0.3 \\
& R(u)=0.5+0.02228485017 t^{0.3}\left(0.2+0.9460233055 t^{0.1}\left(20.85455819 t^{0.1}+0.2\right)\right. \\
& \left.-0.03153411018\left(0.2+0.05886367234 t^{0.1}\right) t^{0.1}\right) \\
& \gamma=0.4 \\
& R(v)=0.5+0.02254120996 t^{0.4}\left(0.2+0.9460233055 t^{0.1}\left(20.85455819 t^{0.1}+0.2\right)\right. \\
& \left.-0.03153411018\left(0.2+0.05886367234 t^{0.1}\right) t^{0.1}\right) \\
& \gamma=0.5 \\
& R(w)=0.5+0.02256758334 t^{0.5}\left(0.2+0.9460233055 t^{0.1}\left(20.85455819 t^{0.1}+0.2\right)\right. \\
& \left.-0.03153411018\left(0.2+0.05886367234 t^{0.1}\right) t^{0.1}\right)
\end{aligned}
$$

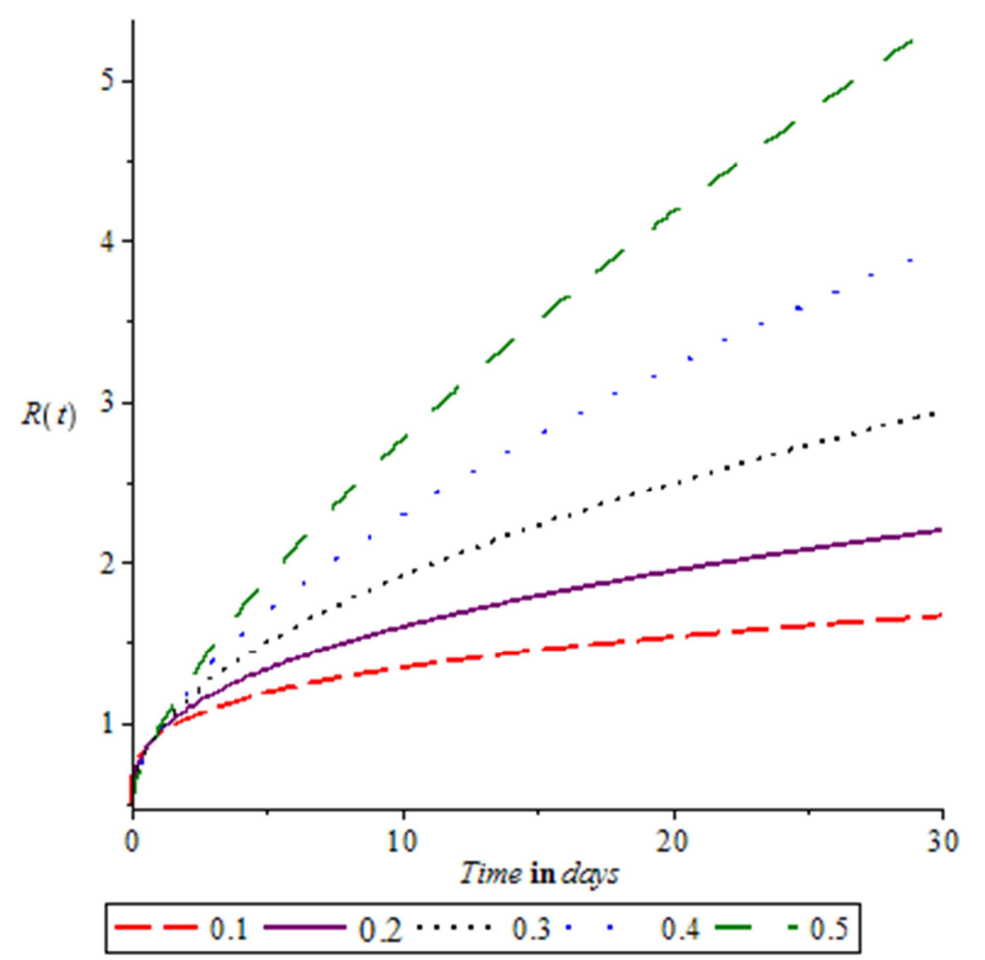

Figure 3. Plot of numerical solution of Recovered class $R(t)$ corresponding to different timein thirtydays.

\section{Conclusion}

In this paper, the framework for modeling and numerical analysis of infectious diseases was carried out using the extended Laplace Adomian Decomposition method for its analysis. Three classes were presented in the model. Namely: the susceptible class $S$, the infected population $I$ and the recovered population from the infection $R$. From the graphical results it is clear that the result obtained by using
LADM is very efficient. It also shows that the method used can predict the behavior of the different variables accurately for the domain under investigation. It also

Indicates that the efficiency of this method can be increased by increasing the terms.

The dynamics of various domains have been indicated in Figure 1, Figure 2, and Figure 3, respectively.

The result also shows that the susceptible class increases as the value of $\gamma$ increases. This is also applicable to the 
other classes considered in this paper.

\section{References}

[1] Wu J. T, Leung K, Leung GM (2020). Now casting and forecasting the potential domestic and international spread of the 2019-nCoV outbreak originating in Wuhan, China: a modeling study. Lancet; 395: 689-97.

[2] Rui-Zing M, Jiming L, William K. W, Cheung, Xiang W. (2017). Stochastic modelling of infectious diseases for heterogeneous populations. Infectious diseases of poverty. 5 (107): 1-11.

[3] Gurav YK, Pawar SD, Chadha MS, Potdar VA, Deshpande AS, Koratkar (2010). Pandemic influenza A (H1N1) 2009 outbreak in a residential school in Panchgani, Maharashtra, India. Indian J Med Res; 132: 67-71.

[4] Sattenspeiel L, Herring DA (2003).. Simulating the effects of quarantine on the spread of 1918 flu in central Canada. Bull Math Biol; 65: 1-26.

[5] Shil P, Bidaye S, Vidyasagar PB (2008). Analyzing the effects of surface distribution of pores in cell electroporation for a cell membrane containing cholesterol. J Phys D: Appl Phys; 41: 551-557.

[6] Deguen S, Thomas G, Chau NP (2000). Estimation of the contact rate in a seasonal SEIR model: application to chickenpox incidence in France. Statist. Med.; 19: 1207-1216.

[7] Wang J, McMichael AJ, Meng B, Becker NG, Han W, Glass K, Wu J, Liu X, Liu J, Li X, Zheng X (2006). Spatial dynamics of an epidemic of severe acute respiratory syndrome in an urban area. Bull. World Health Organization; 84 (12): 965 968.

[8] Kongnuy R, Pongsumpun P 2011. Mathematical modeling for Dengue transmission with theeffect of season. Int. $J$ Biological Life Sci; 7 (3): 143-147.

[9] Constatenos S., Cle A, Lucia R. Christos G, Elefterios M. (2015). Modeling the 2014 Ebola Virus Epidemic - AgentBased Simulations, Temporal Analysis and Future Predictions for Liberia and Sierra Leone. PLoS Currents. 2015: 1-18.

[10] Ruan S, Xiao D, Beier JC (2008). On the Delayed RossMacdonald Model for MalariaTransmission. Bull Math Biol. 70 (4): 1098-1114.

[11] Massad E, Coutinho FAB, Burattini M. N, Amaku M (2010). Estimation of $R$ from the initial phase of an 0 outbreak of a vector borne infection. E. Tropical Medicine Intl Health.; 15 (1): $120-126$.

[12] Adomian G, (1988). A Review of the Decomposition Method in Applied Mathematics, J. Math. Anal. Appl. 135: 501-544.

[13] Somali S. and Gokmen G. (2007). Adomian Decomposition Method for Non-Linear Sturm-Liouville Problems, Surveys in Mathematics and its Applications, Vol. 2, 11-20.

[14] Adomian G., Adomian G. E. (1984). A global method for solution of complex systems, Math. Model. 5521-568.

[15] Adomian G. (1994). Solving Frontier Problems of Physics: The DecompositionMethod, Kluwer Academic Publishers, Dordecht.

[16] Wazwa A. M. (2000). A new algorithm for calculating Adomian polynomials for non-linear Operators, Appl. Math. Comput. 111: 53-69. 